\title{
Pressure dependency on a nanosecond pulsed dielectric barrier discharge plasma actuator
}

Cite as: Phys. Plasmas 26, 063512 (2019); https://doi.org/10.1063/1.5092703

Submitted: 13 February 2019 . Accepted: 03 June 2019. Published Online: 18 June 2019

Michael M Wojewodka, Craig White, Takahiro Ukai, Andrew Russell, and Konstantinos Kontis (D)

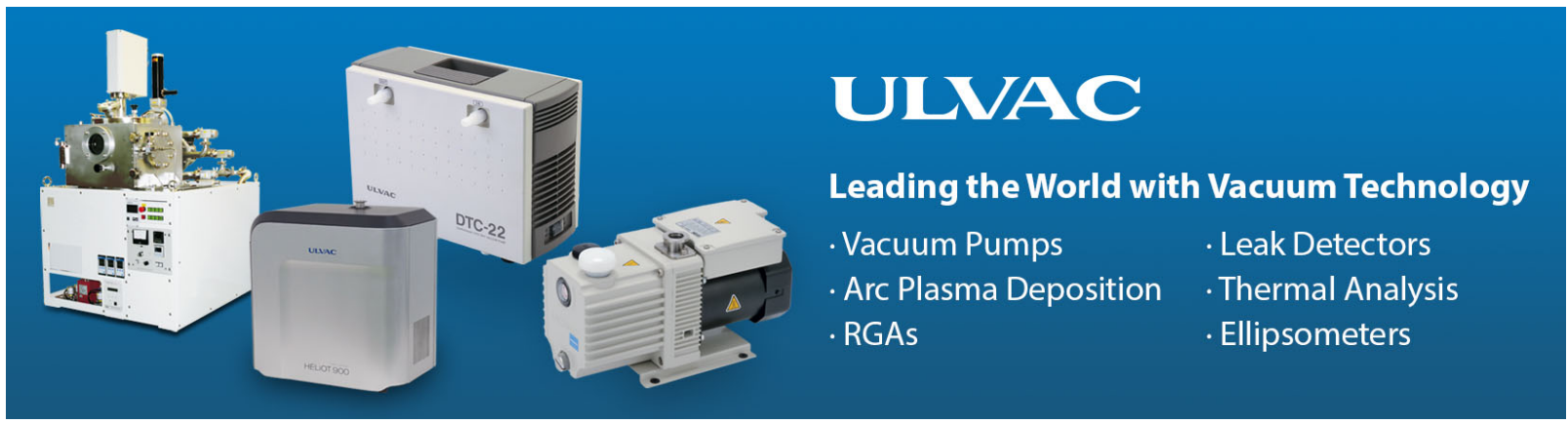




\title{
Pressure dependency on a nanosecond pulsed dielectric barrier discharge plasma actuator
}

\author{
Cite as: Phys. Plasmas 26, 063512 (2019); doi: 10.1063/1.5092703 \\ Submitted: 13 February 2019 - Accepted: 3 June 2019 . \\ Published Online: 18 June 2019
}

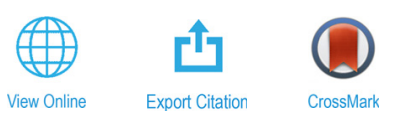

Michael M Wojewodka,, ${ }^{1, a)}$ Craig White, ${ }^{1, b)}$ Takahiro Ukai, $^{2, b)}$ Andrew Russell, ${ }^{1, b)}$ and Konstantinos Kontis ${ }^{1, b)}$ (iD

\author{
AFFILIATIONS \\ ${ }^{1}$ School of Engineering, Aerospace Sciences, University of Glasgow, Glasgow G12 8QQ, United Kingdom \\ ${ }^{2}$ Department of Mechanical Engineering, Osaka Institute of Technology, Osaka 535-8585, Japan \\ ${ }^{a}$ Author to whom correspondence should be addressed: m.wojewodka.1@research.gla.ac.uk

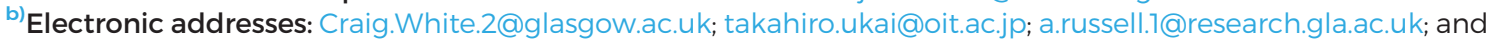 \\ Kostas.Kontis@glasgow.ac.uk
}

\begin{abstract}
The behavior of a nanosecond pulsed dielectric barrier discharge (ns-DBD) plasma actuator with the ambient pressure from 30 to $100 \mathrm{kPa}$ was characterized with Schlieren images. Shock wave propagation speed and strength were recorded, showing clear trends with decreasing ambient pressure. Higher ambient pressures result in stronger shock waves; this has been observed irrespective of the actuator thickness. This might be explained with fewer air molecules to ionize at lower ambient pressures and hence a lower temperature from the exothermal recombination reactions. The thickness of the dielectric barrier also influences the shock strength. In accordance with previous findings, it was confirmed that a thinner dielectric barrier results in a greater shock strength. NS-DBD shock waves were modeled numerically using OpenFOAM through a source term added to the energy equation, which controls the amount of thermal energy added to the near-wall deposition region. The compressible, unsteady sonicFoam solver was used with second order schemes. A mesh sensitivity study gives confidence that the solution is grid independent. The overall shock wave structure and propagation speed match well with experimental data. The heat addition required to reproduce experimental results varied with ambient pressure. Less heating of the near-wall region was needed with lower ambient pressures.
\end{abstract}

Published under license by AIP Publishing. https://doi.org/10.1063/1.5092703

\section{INTRODUCTION}

A typical dielectric-barrier-discharge (DBD) plasma actuator (PA) consists of two electrodes, one exposed to the free stream and one covered by a dielectric layer, ${ }^{1}$ see Fig. 1. Dependent on the signal fed to the electrodes, two distinct characteristics of DBD plasma actuation are common: alternate-current (ac) and nanosecond (ns) - with different operating principles and mechanisms to influence the flow.

For the ns-DBD PA operation, the electrodes are fed with very high voltage nanosecond pulses. During each pulse, the gas between the electrodes is ionized and charged particles are attracted to the high voltage electrode, traveling along the dielectric as close to the electrode as possible. ${ }^{2}$ As the pulse ends, the electrons are released moving in a direction opposite to the relative position of the high voltage electrode. Plasma streamers are formed and induce local changes to the temperature, density, and viscosity fields. Ultrafast gas heating occurs in the region of plasma streamers ${ }^{3}$ which dictate the effective heating region. The highest temperature is generated locally at the exposed electrode edge. As the streamer propagates along the dielectric, it is accompanied by a surface heating rise.
Electrons collide with air particles with enough energy to dissociate them. Their recombination is an exothermal process releasing large amounts of thermal energy ${ }^{6}$ over very short time periods (about $1 \mu \mathrm{s}$ ). This increases the local volume temperature by an approximate factor of ten. The temperature increase of hundreds of degrees Kelvin in a few dozen nanoseconds was recorded. ${ }^{8-11}$ Due to the short time frame, the gas does not expand, resulting in the increase in both pressure and temperature. The gas expands adiabatically generating a shock wave. The main mechanism of impact is the energy transfer to the near-wall gas in the form of a heat plume, ${ }^{5,7,12}$ which results in a shock wave. Strong local changes in temperature and viscosity accompanied by shock waves are the main method of interaction of the ns-DBD plasma actuator with the flow. This offers both a very high flow control authority potential and low power consumption. ' Unlike ac-DBD plasma actuators, there is very weak ionic wind/momentum generated by ns-DBD plasma actuation. ${ }^{13}$

The effect of geometry on shock wave strength was investigated in a previous study ${ }^{14}$ with the results indicating that a thinner dielectric results in a stronger shock wave for a given voltage and pulse 


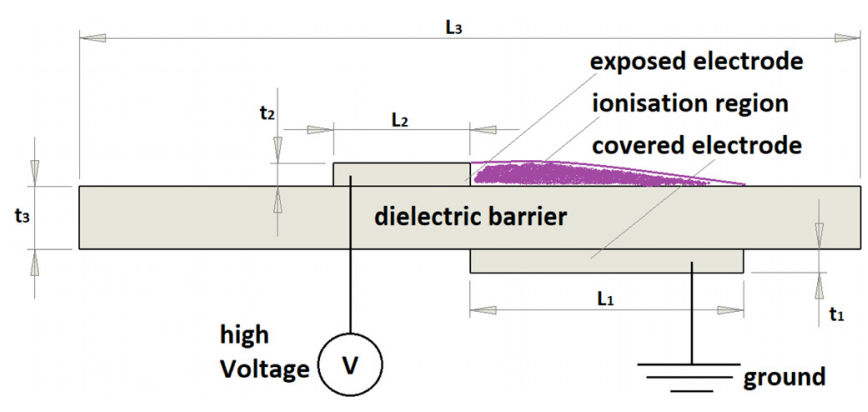

FIG. 1. Schematic representation of the typical dielectric barrier discharge plasma actuator configuration for ac and ns operation.

frequency input. This was also shown in studies by Van den Broecke and Avallone. ${ }^{15,16}$

With the general processes governing the formation of ionized plasmas established through benchmark studies, ${ }^{1,7,17-19}$ the attention of many researchers moved to characterizing plasma actuators at various conditions and classifying their suitability as a flow control application in a number of environments.

Thermal effects of ns-DBD actuators and more specifically their ability to mitigate aircraft icing were investigated by Lui and Chen. ${ }^{20,21}$ Winkel and others ${ }^{15,22}$ looked at the energy deposition of plasma actuators. Do Nascimento et al. ${ }^{23}$ investigated the behavior of DBD actuators in different gasses using argon (Ar), helium (He), and nitrogen $(\mathrm{N})$, while helium was also used by Nastuta and Liu. ${ }^{24,25}$ A novel method to measure the temperature field was designed and tested on ns-DBD plasma actuators by Ukai et al. ${ }^{26}$

Pressure dependency of ac-DBD plasma actuators was studied by Valerioti and Corke ${ }^{27}$ for pressures from 17 to $900 \mathrm{kPa}$ and others. ${ }^{28-30}$ Their studies conclude that the body force produced by ac-DBD actuators has two maxima-one at subatmospheric pressures and the other at superatmospheric pressures. These peaks depend on the voltage input and occur at pressures further away from atmospheric conditions with increasing voltage input levels.

However, there is a lack of data on pressure and temperature dependency of ns-DBD plasma actuators. This study aims at characterizing ns-DBD actuation with varying ambient pressures for subatmospheric cases for pressures of 30 to $100 \mathrm{kPa}$. The effects of pressure dependency on shock wave generation by ns-DBD plasma actuation were investigated experimentally and numerically. The ns-DBD plasma actuator is operated in a vacuum chamber, and Schlieren photography is used for shock wave visualization.

\section{EXPERIMENTAL SETUP}

Experiments were conducted at the University of Glasgow using the same equipment and basic setup as those used by Ukai et al. ${ }^{26}$ who used the same facility for their research.

The ns-DBD PA used in the study is shown in Fig. 2. The exposed high-voltage and ground electrodes were 5 and $10 \mathrm{~mm}$ wide, respectively. The electrodes were made of $35 \mu \mathrm{m}$ copper. The electrodes are separated by a dielectric material known as glass reinforced epoxy (GRE FR-4: Flame Retardant Type 4) with thicknesses of 0.4, 0.8 , and $1.6 \mathrm{~mm}$. There is no discharge gap between the electrodes. The lateral length of all electrodes is $85 \mathrm{~mm}$. The ground electrode was fully covered by three layers of polyimide film, approximately $210 \mu \mathrm{m}$ in total. The polyimide film covered the exposed electrode $7.5 \mathrm{~mm}$ from both lateral side edges to prevent plasma arcing on the sides of the actuator.

The actuators were made using double-sided photolithography. The masks, shown in Fig. 2, for the process were created using CAD software. The copper comes with a photoresist coating, which is exposed under UV light for $30 \mathrm{~s}$. It is then submerged in a developer bath that removes the resist that was exposed to the UV light. The

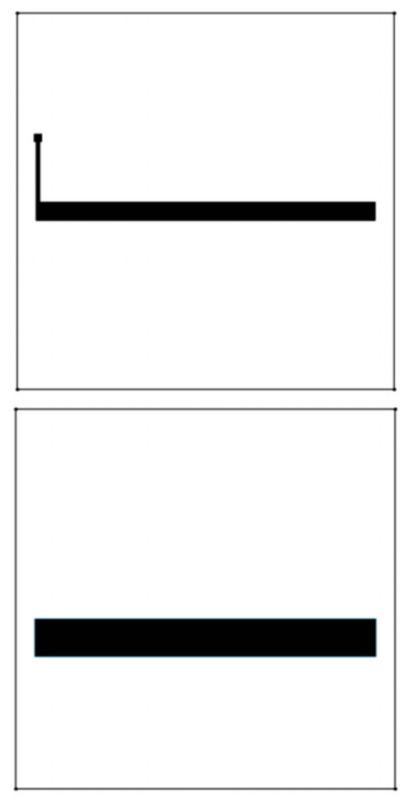

(a)

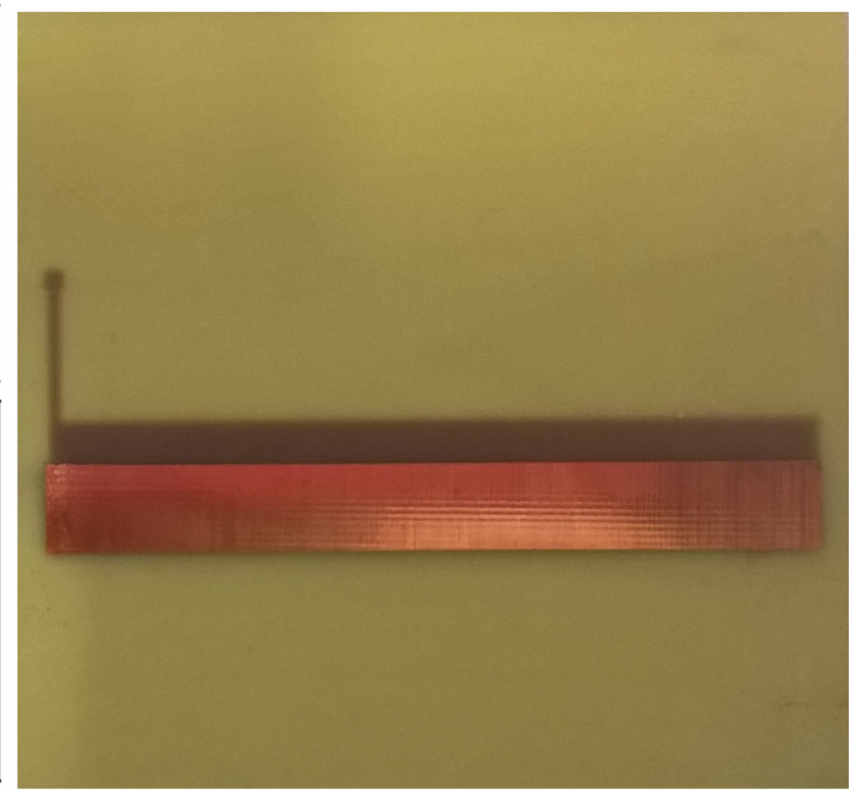

(b)
FIG. 2. Example of the actuator used (not to scale): masks used for the etching process [2(a)]. Top: exposed, high voltage electrode. Bottom: covered, ground electrode. Image of the actuator [2(b)] made of the GRE dielectric with copper electrodes. 
developer is Seno 4006, a solution of Potassium hydroxide, and Disodium metasilicate. The board is next sprayed with a ferric chloride solution to remove the exposed copper. The whole process is usually concluded in about $1 \mathrm{~min}$ for each side of the actuator. Once the copper is removed, the remaining photo resist is cleaned with acetone.

The discharge is driven by a high-voltage nanosecond pulse generator [Megaimpulse, model: NPG18/3500(N)] that supplies negative pulse polarity at a pulse rise time of approximately $4 \mathrm{~ns}$. In the present study, an input voltage of $12 \mathrm{kV}$ (negative polarity) was used with a pulse frequency of $1 \mathrm{kHz}$ controlled by a function generator (AIM \& THURLBY THANDAR INSTRUMENTS, model: TG2000). The negative polarity produces large gross energy related to strong gas heating, ${ }^{31}$ and thus, negative polarity was used in this study. The supplied high-voltage pulses were transferred from the pulse generator to the exposed electrode by a $75 \Omega$ coaxial cable. The ambient temperature was monitored using a K-type thermocouple with a data acquisition module system (National Instruments Corp., model: NI-9213, 24 bit) driven by LabVIEW. The pressure in a quadrilateral, stainless steel vacuum chamber with a volume of $0.138 \mathrm{~m}^{3}$ was controlled through a vacuum pump and was measured through a pressure transducer (Kulite, model XTE-190M, pressure range: 0 to $170 \mathrm{kPA}$ Absolute).

The Schlieren technique with a standard Z-type optical arrangement was employed to visualize the qualitative density gradient above the ns-DBD plasma actuator. The Schlieren system consists of a continuous light source (Newport, model: 66921) with a $450 \mathrm{~W}$ Xe arc lamp, a condenser lens with a focal length of $70 \mathrm{~mm}$, a pinhole, a pair of $203.3 \mathrm{~mm}$ diameter concave mirrors with a focal length of $1829 \mathrm{~mm}$, a circular dot cutoff plate, an imaging lens, and a highspeed camera. The pinhole in front of the condenser lens creates a light spot that illuminates the first concave mirror. The light beam is then collimated by the first mirror. The collimated beam passes through a $137 \mathrm{~mm}$ diameter quartz window of the vacuum chamber and the test section where the ns-DBD PA is located. A second parabolic mirror reflects the beam onto optics and the camera. The circular dot plate is positioned at the focal point of the second mirror.

Two high speed cameras, HPV-1 (Shimadzu) and Fast cam SA 1.1 (Photon), were used to capture the effect of pressure on the shock propagation speed and the shock strength. The images were required at a frame rate of $75 \mathrm{kfps}$ (Photon) and $500 \mathrm{kfps}$ (Shimadzu) with an exposure time of $1 \mathrm{~s}$.

In order to measure the high voltage signal provided by the nanosecond pulse generator, the back-current shunt technique was used. ${ }^{16}$ Due to the high voltage levels experienced through the core of the supply cable, the signal is instead measured from the current generated in the shielding of the coaxial cable. This is done using a series of resistors, connected in parallel to form a shunt resistor. This resistor is placed across a break in the ground of the coaxial cable and the voltage across this shunt resistor is measured. The current generated in the ground connection can be related to the voltage using Eq. (1) from transmission line theory

$$
V_{\text {supply }}=\frac{L_{\text {characteristic }}}{I},
$$

where $\mathrm{V}_{\text {supply }}$ is the supply voltage, $\mathrm{I}$ is the current through the transmission line, and $\mathrm{L}_{\text {characteristic }}$ is the characteristic impedance of the transmission line. For the coaxial cable used in this work, the characteristic impedance is $75 \Omega$. The resistance of the shunt resistor is designed to be low, on the order of milliohms in order for the generated voltage to be measured directly through an oscilloscope. The voltage measured can then be converted to the actual voltage supplied to the DBD plasma actuator using the following equation:

$$
V_{\text {actual }}=V_{\text {shunt }} \cdot \frac{L_{\text {characteristic }}}{R_{\text {shunt }}},
$$

where $\mathrm{V}_{\text {shunt }}$ is the voltage measured across the current shunt and $\mathrm{R}_{\text {shunt }}$ is the known current shunt resistance.

\section{NUMERICAL METHODS}

A phenomenological model of ns-DBD plasma actuators is used in this study. The model is adapted from the method proposed by Gaitonde $^{32,33}$ and is based on a heated volume region. Similar approaches are used by Zhao and Kinefuchi. ${ }^{19,34}$ Other researchers chose to create a more physical model of ns-DBD plasma including near wall electric field equations, electron and ion densities in the gas, and drift diffusion equations. ${ }^{35,36}$ Unfer and Boeuf ${ }^{4}$ implemented a two dimensional ns-DBD model taking into account the drift diffusion and local field approximations. The latter is used to approximate the energy gained by charged particles from the electric field by locally balancing the gains with losses due to collisions with neutral molecules. The former assumes that a particle's momentum can be estimated by equating particle's fluxes with the sum of a drift and a diffusion term. However, most researchers agree that given the timescales involved, there is no added benefit from adding ion motion, electron diffusion, and chemical recombination processes to plasma modeling.

A numerical model of ns-DBD PA was implemented in OpenFOAM for the use with transient, density-based computational fluid dynamics (CFD) calculations. A region of the mesh is selected for which a Gaussian temperature profile is prescribed to simulate the heat addition by the ns-DBD plasma actuators. See Fig. 3 for the temperature profile prescribed to the heated region. The nonsymmetric nature of the profile results in both a cylindrical and planar shock wave being generated. The length of the Gaussian profile was chosen based on the covered electrode width along which the plasma is generated. The discharge distance and streamer length, which actually

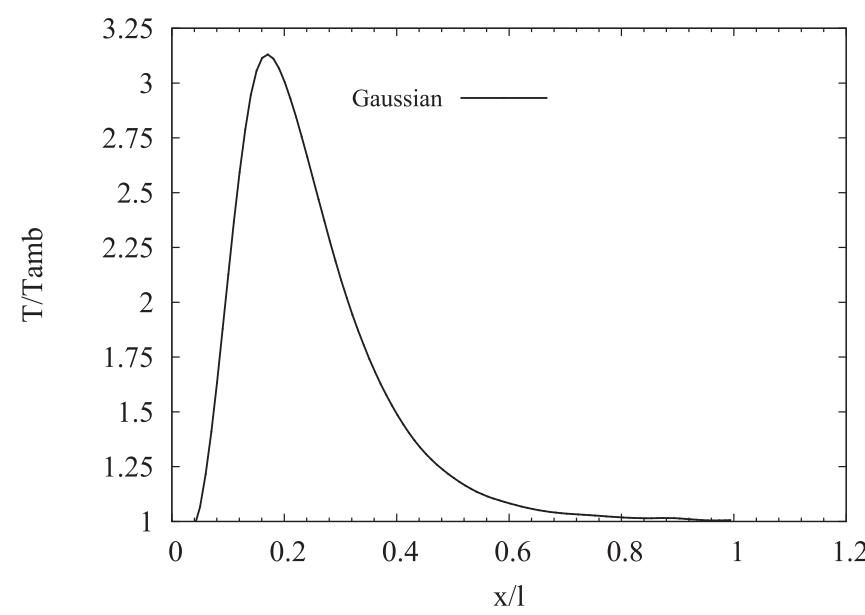

FIG. 3. Gaussian distribution of the temperature profile prescribed to the heated region. Shape adapted from Gaitonde. 
govern the extent of the heated region, were not available from experiments to use.

A source term in the energy equation is added to control the amount of heat added to the selected volume. The Gaussian temperature profile is essential to capture the shock structure while the uniform and step profiles of temperature do not produce a good match. ${ }^{32}$ In the current implementation, the pulse time, rest time, actuator length and width, and its starting point can all be set by the user. It is also possible to create multiple heated regions and investigate the effect of a number of actuators. The governing equation defining the Gaussian profile is added to a factor $(\chi)$ of the ambient temperature

$$
T(x)=T_{a m b}+\sqrt{\frac{\lambda}{2 \pi x^{3}}} \exp \left(-\frac{\lambda}{2 \mu^{2} x}(x-\mu)^{2}\right) T_{a m b} \chi .
$$

The symbols $\lambda$ and $\mu$ are parameters used to alter the Gaussian profile and its mean value. In this study, $\lambda$ is set to 1 and $\mu$ to 0.3 .

For this study, the sonicFoam solver in OpenFOAM was used due to its transient, compressible features. Calculations were performed with second order (Gauss limitedLinear) schemes and the pimple algorithm in laminar, quiescent conditions. The time step was $2 \mathrm{~ns}$ with the pulse time of the ns-DBD model set to $100 \mathrm{~ns}$. The simulation was performed for a total of $60 \mu \mathrm{s}$ with a write interval of $2 \mu \mathrm{s}$. Initially, a mesh independence study was conducted to ascertain that simulation results would not be affected by mesh density. The ambient temperature was set to $293 \mathrm{~K}$, corresponding to the ambient temperature during the experimental campaign.

\section{RESULTS}

The voltage supplied to the actuators is shown in Fig. 4 which also shows the $20 \%$ and $80 \%$ threshold lines. Those were used to evaluate the pulse width, rise time, and fall time of the signal. Measurements show a triangular signal with a pulse width of $6 \mathrm{~ns}$, a rise time of $3.8 \mathrm{~ns}$, and a peak, negative polarity voltage of $9.45 \mathrm{kV}$. The fall time seems to be longer than the rise time lasting $9 \mathrm{~ns}$ based on the $20 \%$ and $80 \%$ threshold values of the peak voltage. Current measurements were not taken during experiments.

The ns-DBD actuator generates a distinct, cylindrical shock wave with a tail which propagates from the actuator surface as is evident from the Schlieren images. There is also a clear planar wave which has been previously recorded in an experiment by Takashima. ${ }^{18}$ Figure 5 shows these two waves for the case of an ambient atmospheric pressure and temperature within the experimental domain and an actuator thickness of $0.8 \mathrm{~mm}$. The shock wave is shown two times after actuation: $13.3 \mu$ s and $26.7 \mu$ s, respectively.

\section{A. Shock propagation}

Propagation speed of the shock wave is calculated based on the location of the shock front in consecutive Schlieren images and the time between these images. The location of the shock front is estimated by analyzing the profile of the image intensity values across it and then searching for the maximum value in a purpose written postprocessing code.

The following six plots, Figs. 6-11, show the shock propagation speed and the distance traveled by the shock front with time for the three actuator thicknesses $(\mathrm{t}=0.4,0.8$, and $1.6 \mathrm{~mm})$.

\section{B. Shock strength}

The shock strength was estimated based on the intensity values of the captured Schlieren images. Image processing software was used to combine a number of Schlieren images showing the propagation of a single shock wave in one image. Next, a purpose written code analyzed the intensity of each pixel on the image along a vertical line from the center of the actuator surface to the top of the image. Peak values

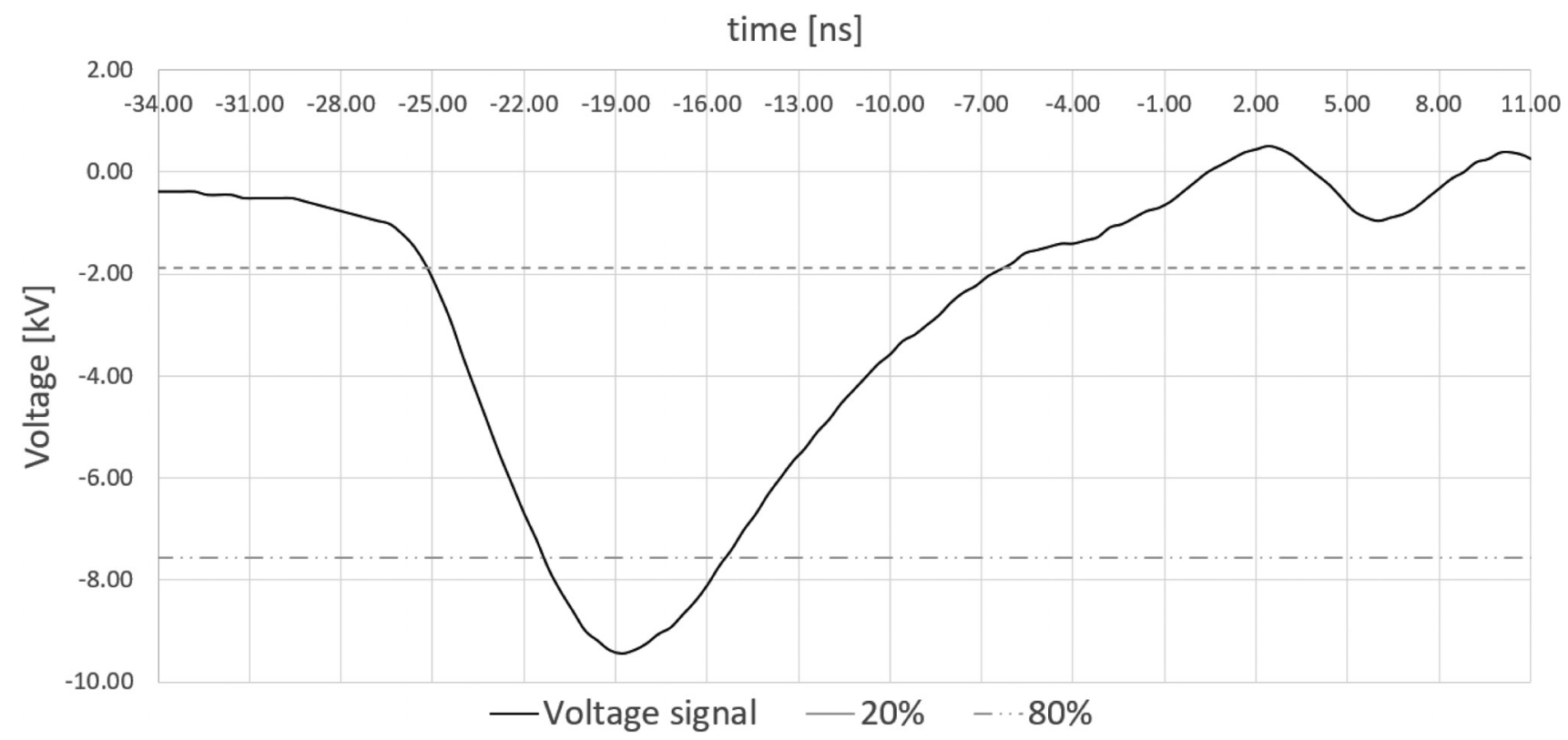

FIG. 4. Voltage signal supplied to plasma actuators with the $20 \%$ and $80 \%$ threshold lines used to evaluate the pulse width, its rise and fall time. 


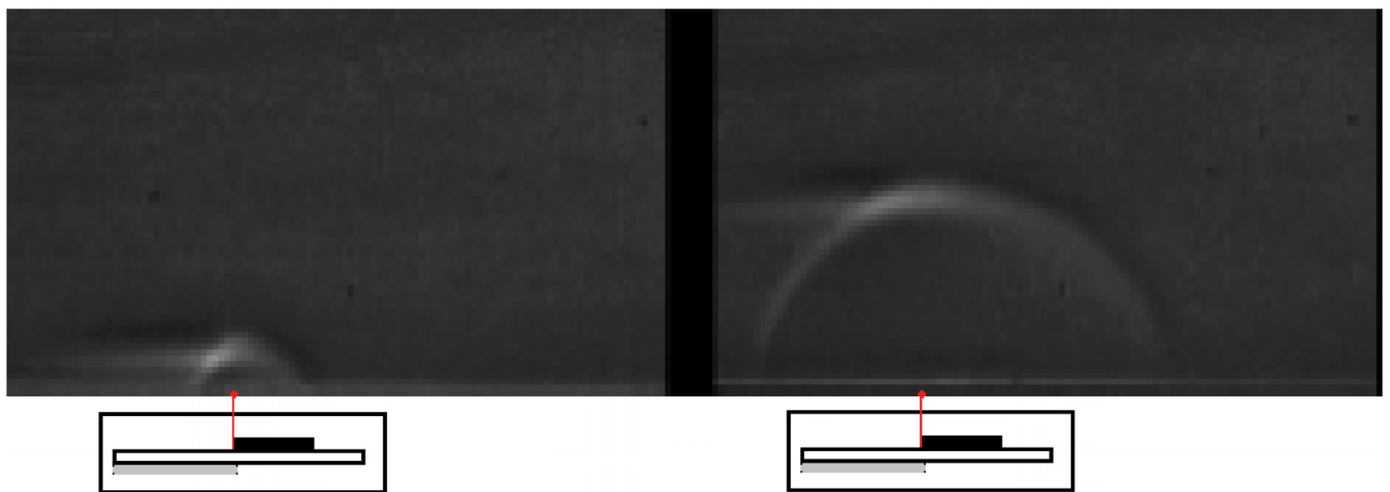

FIG. 5. Schlieren image of distinct, cylindrical, and planar shock waves generated from the ns-DBD actuator of $0.8 \mathrm{~mm}$ thickness at ambient (atmospheric) conditions. The location of the plasma actuator is shown with the ground electrode colored gray, the dielectric barrier colored white, and the high voltage electrode colored black.

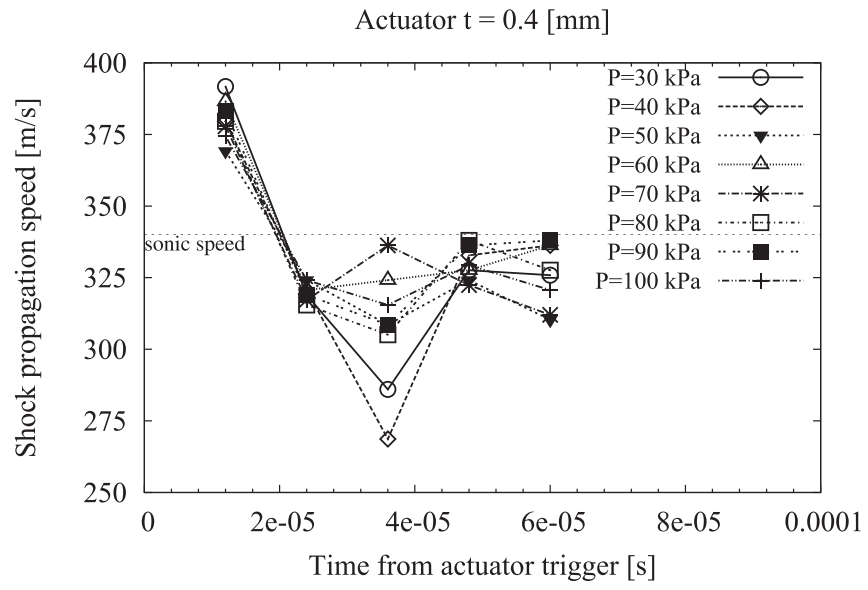

FIG. 6. Shock propagation based on shock front location: time vs velocity. Actuator thickness: $0.4(\mathrm{~mm})$.

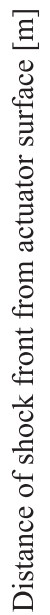

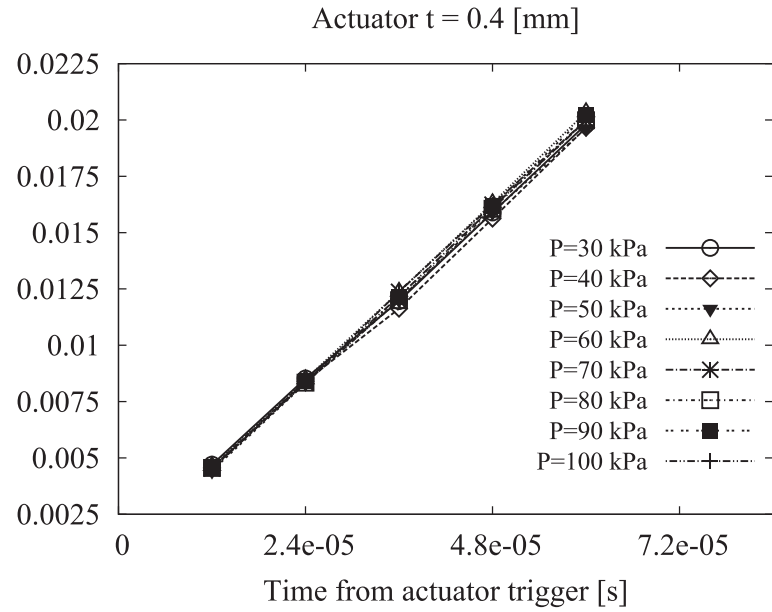

FIG. 7. Shock propagation based on shock front location: time vs distance. Actuator thickness: $0.4(\mathrm{~mm})$.

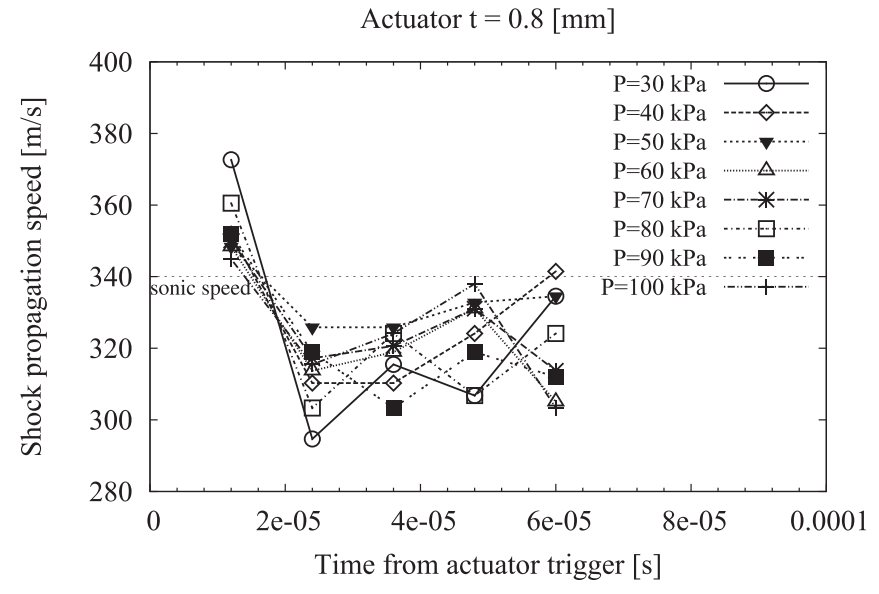

FIG. 8. Shock propagation based on the shock front location: time vs velocity. Actuator thickness: $0.8(\mathrm{~mm})$.

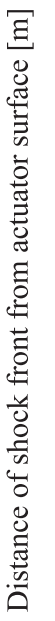

Actuator $\mathrm{t}=0.8[\mathrm{~mm}]$

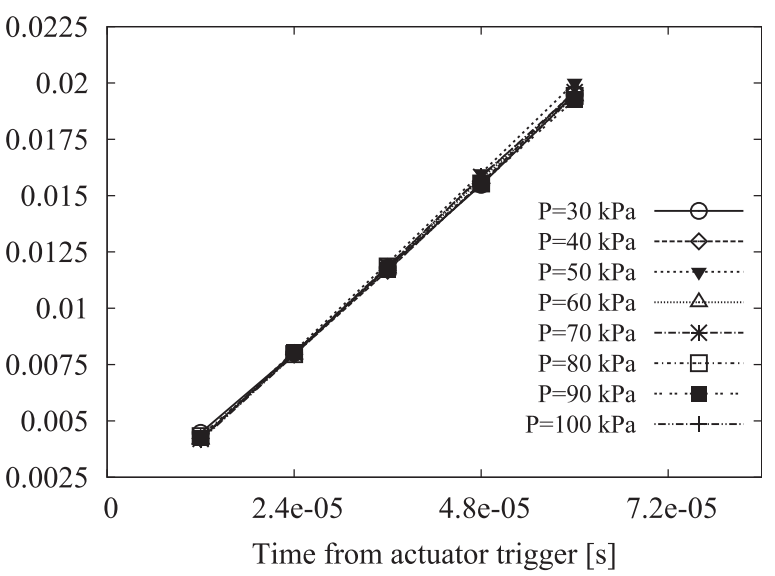

FIG. 9. Shock propagation based on the shock front location: time vs distance. Actuator thickness: $0.8(\mathrm{~mm})$. 
Actuator $\mathrm{t}=1.6[\mathrm{~mm}]$

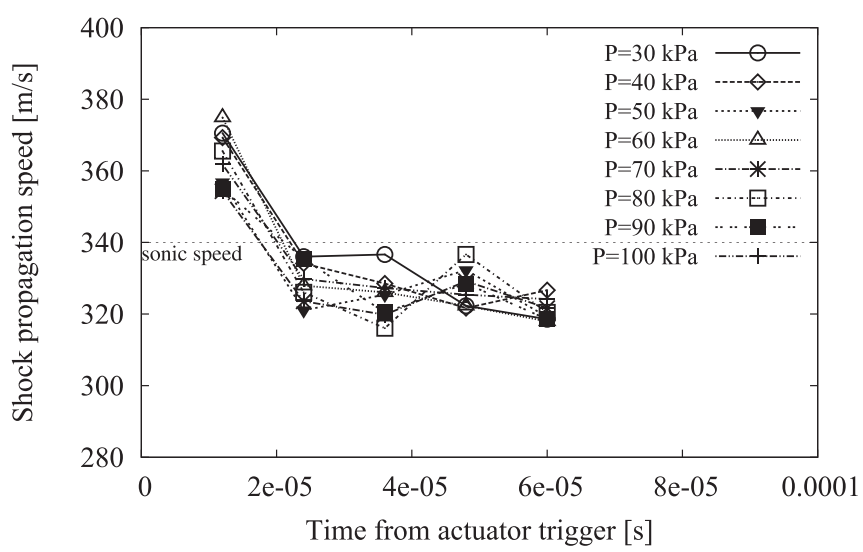

FIG. 10. Shock propagation based on the shock front location: time vs velocity. Actuator thickness: $1.6(\mathrm{~mm})$

of intensities and their corresponding vertical location are also extracted. Peak intensity values, $I$, are normalized by the background intensity, $I b$, of each image. Image intensity was obtained from ten images of shock propagation. Normalized shock intensity, $I / I b$, has a mean value of 5.11 (for a pressure of $60 \mathrm{kPa}$ and a dielectric thickness of $0.4 \mathrm{~mm}$ ) with a standard deviation of 0.243 . The standard deviation is significantly smaller (below 5\%) than the mean, and therefore, there is a high degree in repeatability. An example of this process is shown in Fig. 12.

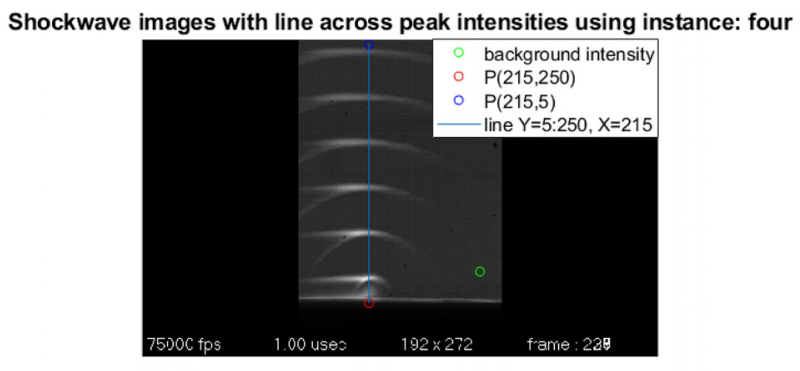

$P(215,5)$ is furthest away from actuator while $P(215,250)$ is closest to actuator

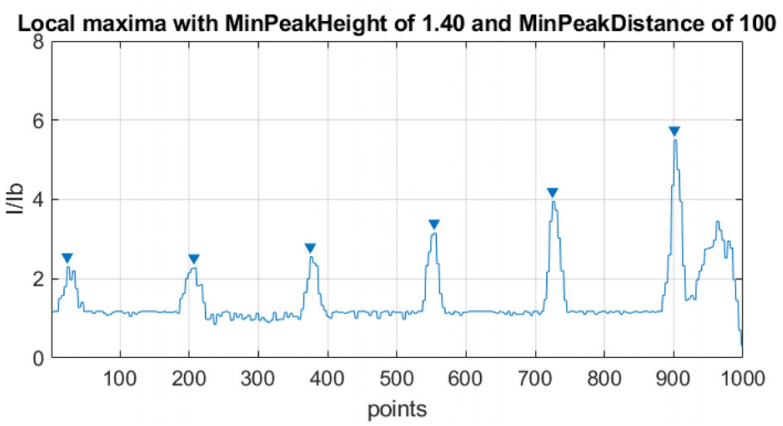

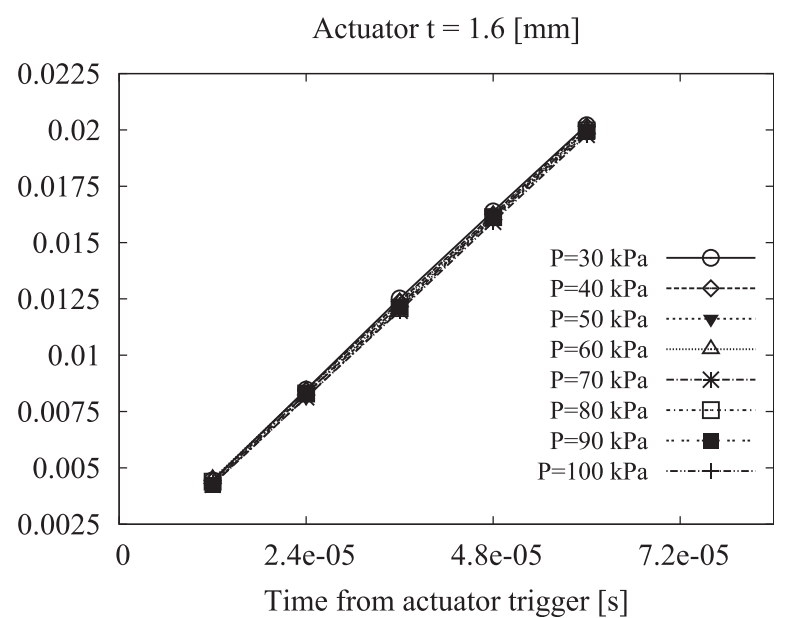

FIG. 11. Shock propagation based on the shock front location: time vs distance. Actuator thickness: $1.6(\mathrm{~mm})$.

Figure 13 shows the intensity profiles of ten instances of recorded shock waves. Profiles indicate little spread in shock strength and wave front speed for recorded shock waves. The intensity values closest to the actuator region, $230<\mathrm{Y}<250$ pixels, show strong gradients in the vicinity of the discharge in what is known as the generated heat plume. ${ }^{6}$

For the three actuator thicknesses used, 0.4, 0.8, and $1.6(\mathrm{~mm})$, the process above was repeated for Schlieren images capturing ten
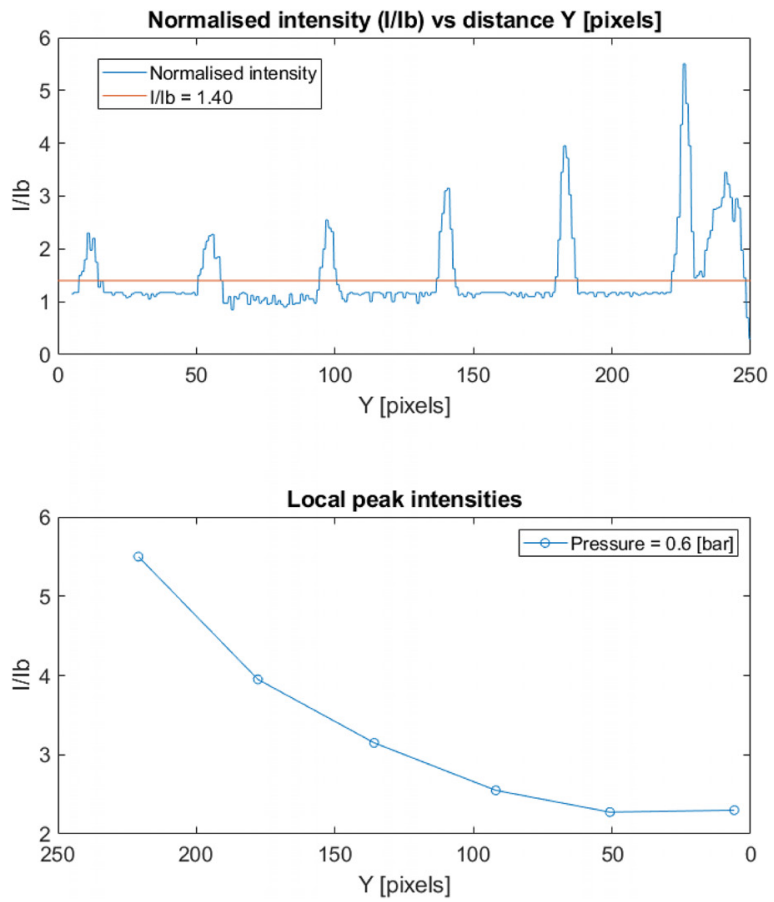

FIG. 12. Example of output from the Matlab code. Top left: Combined Schlieren images of the propagation of a single shock wave with the vertical line (from 215:5 to 215:250), indicating that the position intensities are extracted. Top right: Pixel intensity profile along line from 215:5 to 215:250. Bottom left: Pixel intensity profile with identified peak values that correspond to the shock wave fronts. Bottom right: Normalized shock strength $1 / / b$ vs vertical distance [pixels] from the actuator surface. 


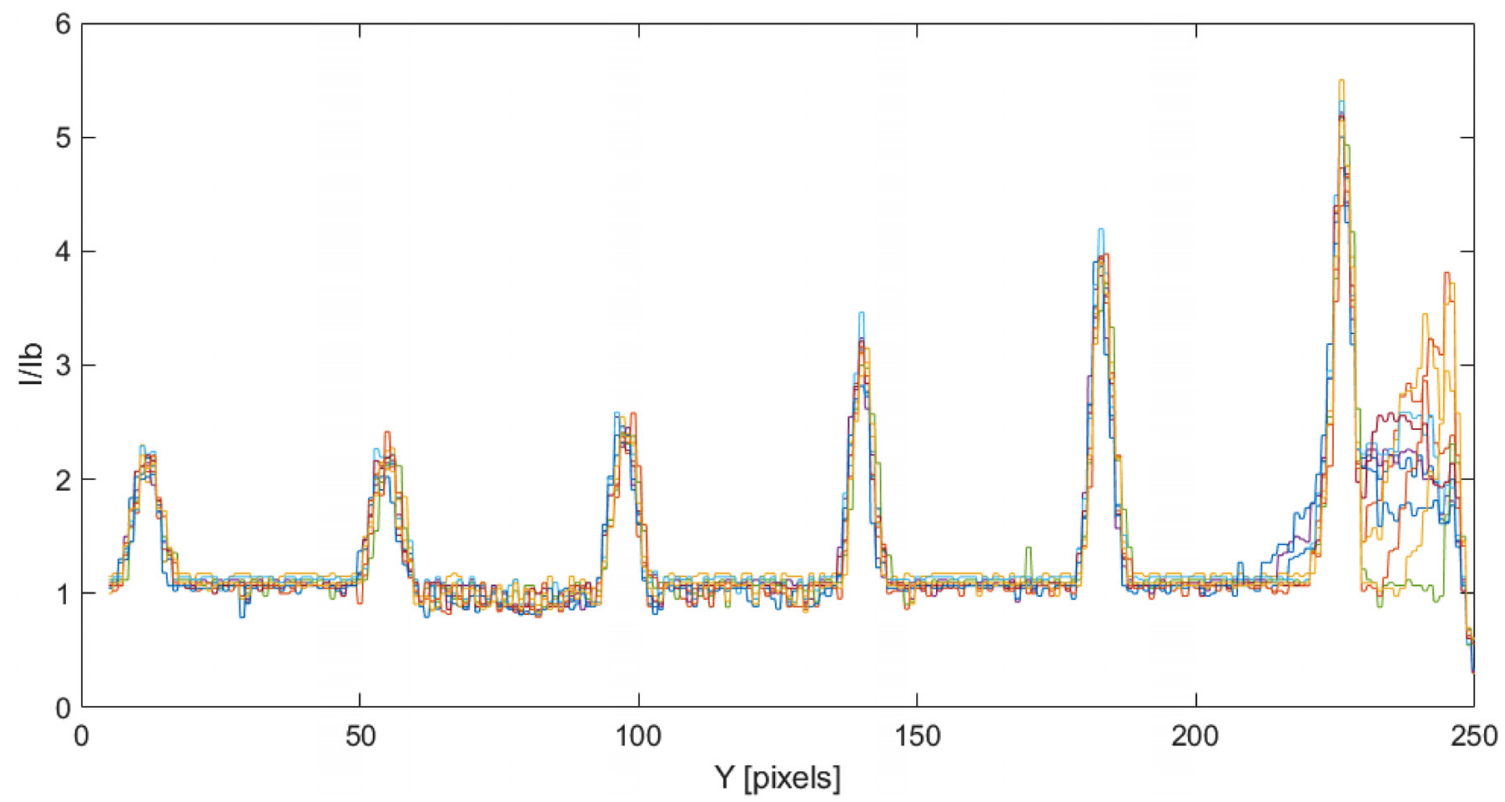

FIG. 13. Normalized intensity profiles, l/lb, along vertical line across ten shock wave propagation images.

separate shock waves for each pressure value in the vacuum chamber. The results presented below are the average of all ten instances. Graphs, in Figs. 14-16, show the normalized shock strength, $I / I b$, vs the vertical distance from the actuator surface. The pressure ranges from 30 to $100 \mathrm{kPa}$.

\section{Numerical ns-DBD model}

The implementation was tested with a simple $2 \mathrm{D}$ case in a quiescent flow. Flow solutions show a shock wave propagating through the domain with the same distinct features observed in the experiment. The results were postprocessed with a purpose written code to determine the exact shock front location at different time steps. Figure 17

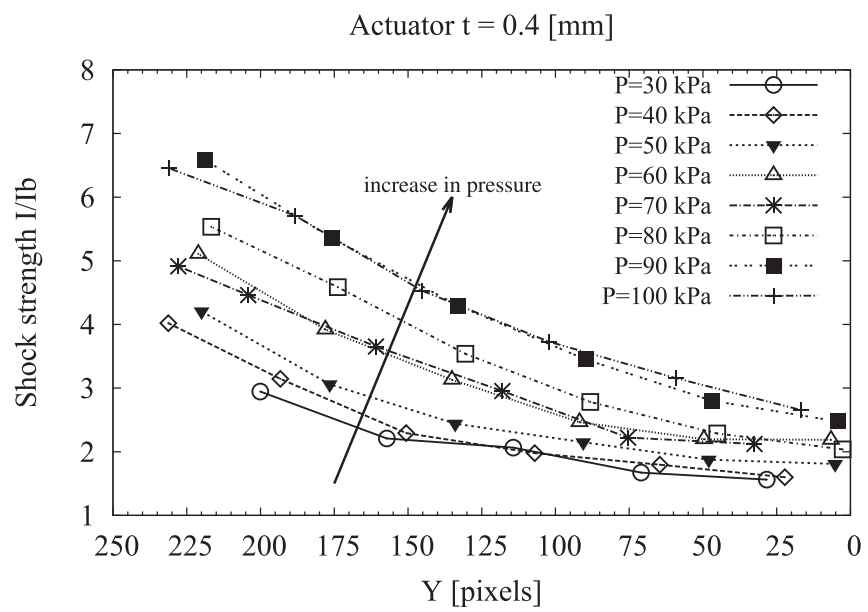

FIG. 14. Normalized shock strength $I / / b$ vs vertical distance from the actuator for a pressure range of 30 to $100 \mathrm{kPa}$. The actuator thickness is $0.4(\mathrm{~mm})$. shows the formed shock wave structure at a time of $10 \mu \mathrm{s}$ after discharge. The figure presents a strong pressure gradient [17(a)] and the density field [17(b)]. The progression of the shock wave front through the numerical domain is depicted in Fig. 18.

The parameter $\chi$ controls the amount of the temperature being added to the heated region. Increasing $\chi$ yields a stronger shock wave with higher velocities (Fig. 19). The model is compared to experimental results obtained. Plots, in Figs. 20-22, show the shock wave propagation speed and distance vs time, for pressures of 30,70 , and $100 \mathrm{kPa}$, respectively.

\section{DISCUSSION}

Previous studies by Roupassov et al. ${ }^{7}$ and Takashima et al. ${ }^{18}$ showed that ns-DBD generated compression waves propagate with an

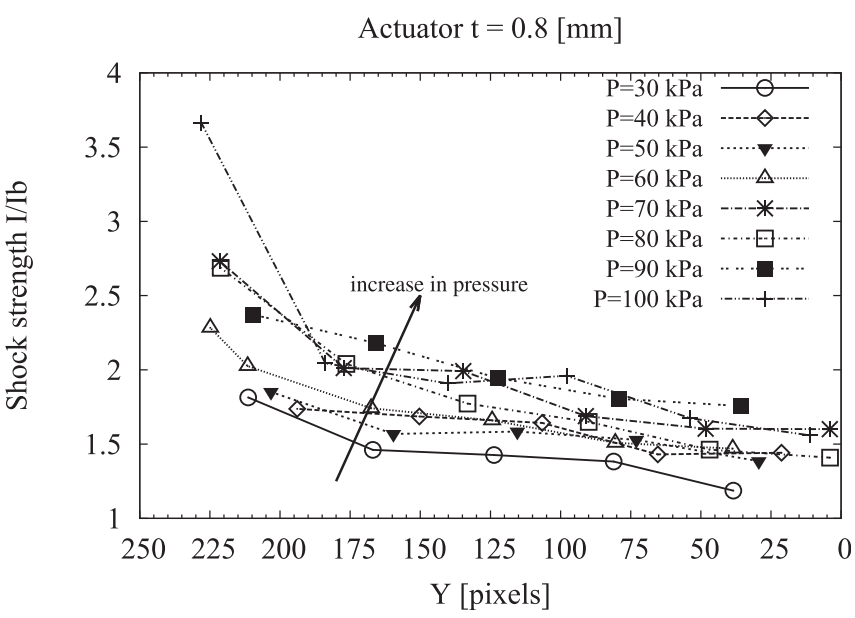

FIG. 15. Normalized shock strength $I / / b$ vs vertical distance from the actuator for a pressure range of 30 to $100 \mathrm{kPa}$. The actuator thickness is $0.8(\mathrm{~mm})$. 


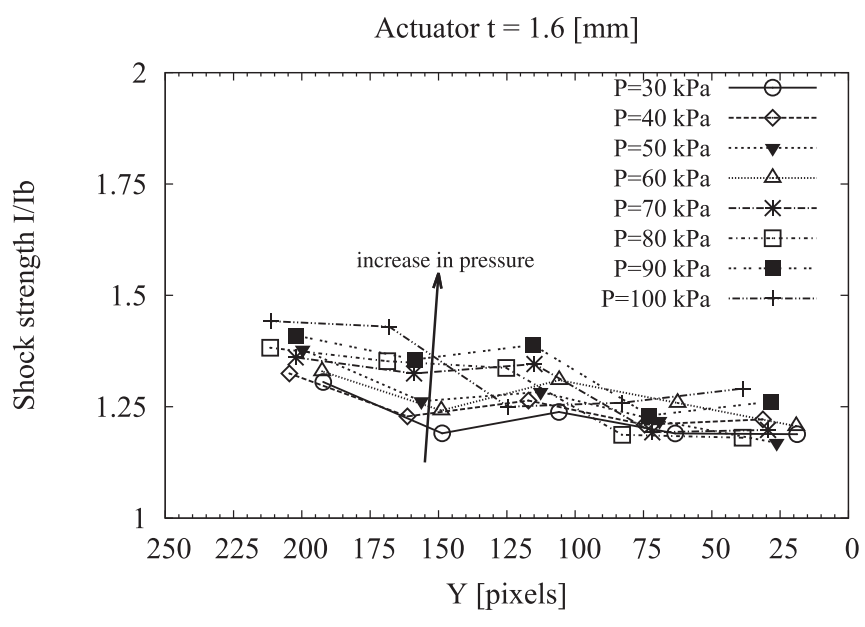

FIG. 16. Normalized shock strength $I / / b$ vs vertical distance from the actuator for a pressure range of 30 to $100 \mathrm{kPa}$. The actuator thickness is $1.6(\mathrm{~mm})$.

approximately sonic velocity. This is confirmed by the shock propagation recorded through Schlieren images in this study as is evident from the constant slope lines in Figs. 7, 9, and 11. These results indicate that there is very little variation for different ambient pressures. However, from Figs. 6, 8, and 10 it is evident that the initial propagation velocity near the discharge is considerably higher though the waves remain sonic. The Mach number increases near the surface due to the high temperature which results in higher wave speeds for Mach $=1$. This has also been noted by other studies. ${ }^{7,18}$

From the results obtained, it is clear that a higher ambient pressure leads to a stronger shock strength. This has been observed for all three actuator thicknesses. With a higher pressure, the air density increases so that there are more particles being ionized by the electric field. Due to the higher number density of particles, it is believed that more exothermal recombination reactions take place which increases the shock strength of the generated shock wave. The effect of ambient pressure on ultrafast gas heating is not clear.

A second observation is that the actuator thickness also influences the shock strength considerably. As the resistance of the electric circuit is proportional to the actuator's thickness, this has a direct effect on the strength of the electric field generated by the ns-DBD PA. The electric field strength determines the rate/extent of the ionization of air particles, and hence, the shock wave strength is enhanced. Therefore, the thinner actuator leads to a greater shock strength.

As is evident from the results, the numerical ns-DBD model captures the overall shock structure and propagation speed accurately. The numerical simulation results compare well with our experiments matching the shock front propagation determined from the Schlieren experiments. The amount of temperature addition required to match experimental results of shock propagation is given in Table I. It outlines the temperature values added to the near wall region for the simulation of ns-DBD generated shock waves. It seems that a lower temperature profile is needed at lower pressure values. This probably means that less flow control authority is available at lower ambient pressures.

\section{CONCLUSIONS}

Shock wave propagation speed and strength were recorded showing clear trends with decreasing ambient pressure. Higher ambient pressures result in stronger shock waves; this has been observed irrespective of the actuator thickness. This might be explained with fewer air molecules to ionize at lower ambient pressures and hence a lower temperature from the exothermal recombination reactions which produce thermal energy. This finding has, potentially, implications on using plasma actuators at altitude, i.e., on airborn vehicles where the ambient pressure is naturally lower than at sea level. However, the effect of ambient temperature would also have to be considered. To the authors' knowledge, no such experiment linking plasma actuator performance to altitude is available in the literature. The data further show that the thickness of the dielectric barrier also influences the shock strength. In accordance with previous findings, it was confirmed that a thinner dielectric barrier results in a greater shock strength.

The overall shock wave structure and propagation speed from CFD match well with our experiments allowing the conclusion that a phenomenological plasma model is sufficient to capture the dominant features of ns-DBD plasma actuators. The heat addition required to reproduce experimental results varied with ambient pressure. Less heating of the near-wall region was needed with lower ambient pressures. This probably means that less flow control authority is available at lower ambient pressures.

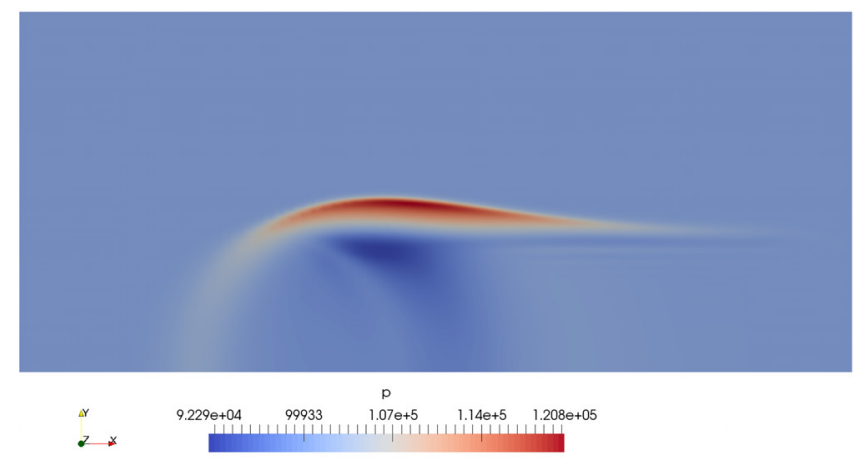

(a)

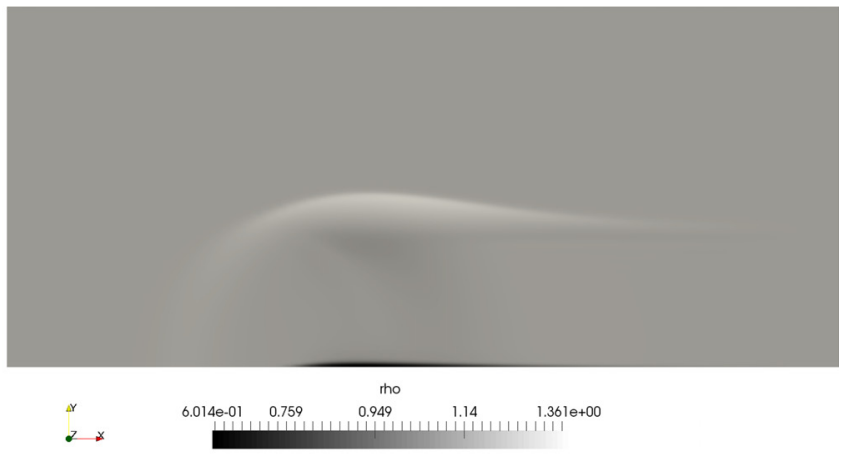

(b)

FIG. 17. Numerically modeled ns-DBD plasma actuator: [17(a)] pressure contours of the shock front and [17(b)] density contours of the shock front. 


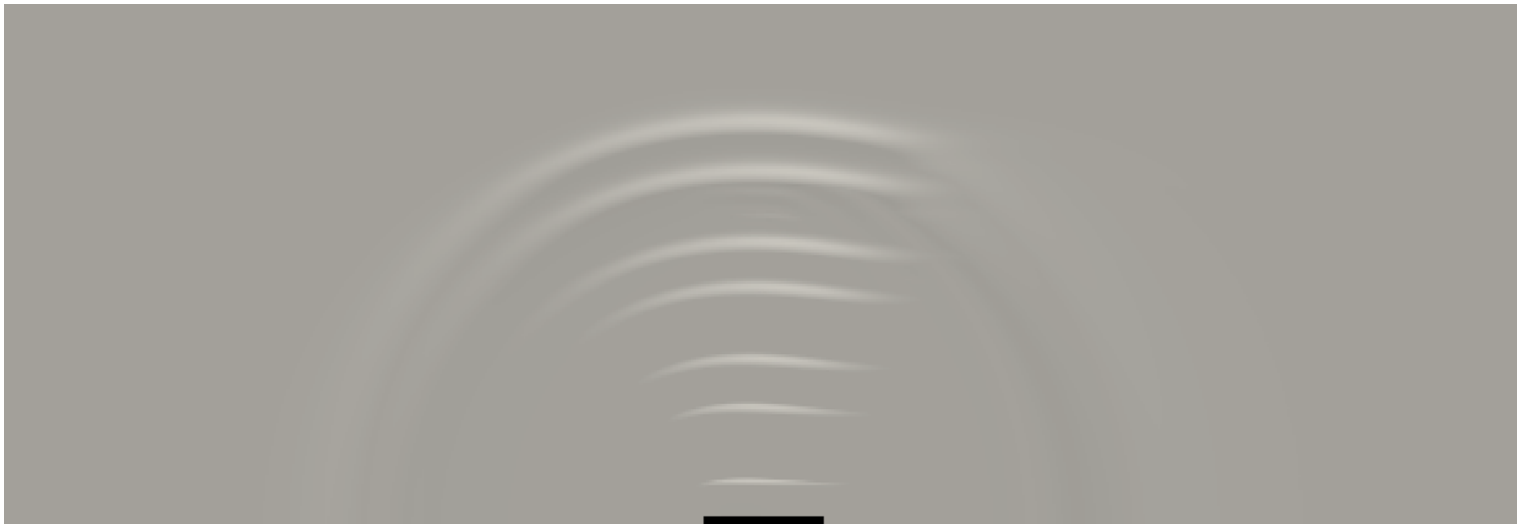

FIG. 18. Progression of shock waves with the ns-DBD model implemented in OpenFOAM. The black colored region at the bottom of the computational domain schematically depicts the heated volume region. This is not to scale. Dimensions of the heated volume are $10 \times 1 \times 10^{-5} \times 1 \mathrm{~mm}$ in the $\mathrm{x}, \mathrm{y}$, and $\mathrm{z}$ directions, respectively.

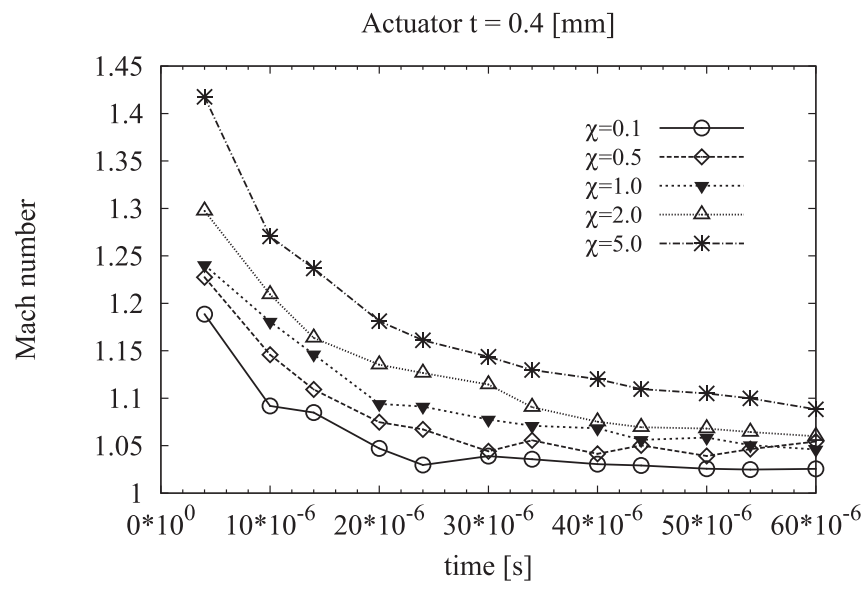

FIG. 19. Effect of parameter $\chi$ on the Mach number of the shock front at ambient conditions.

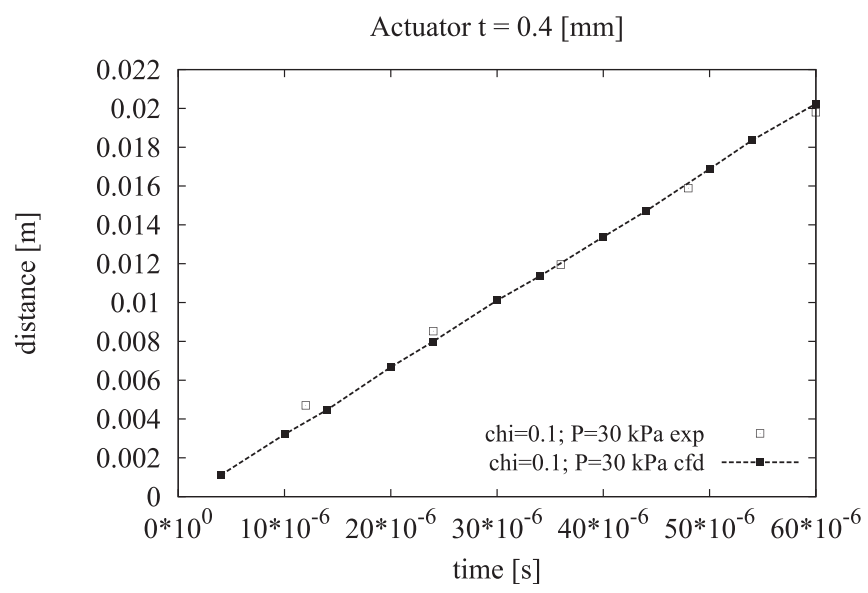

FIG. 20. Comparison of experimental and numerical shock wave propagation for an ambient pressure of $30 \mathrm{kPa}$.

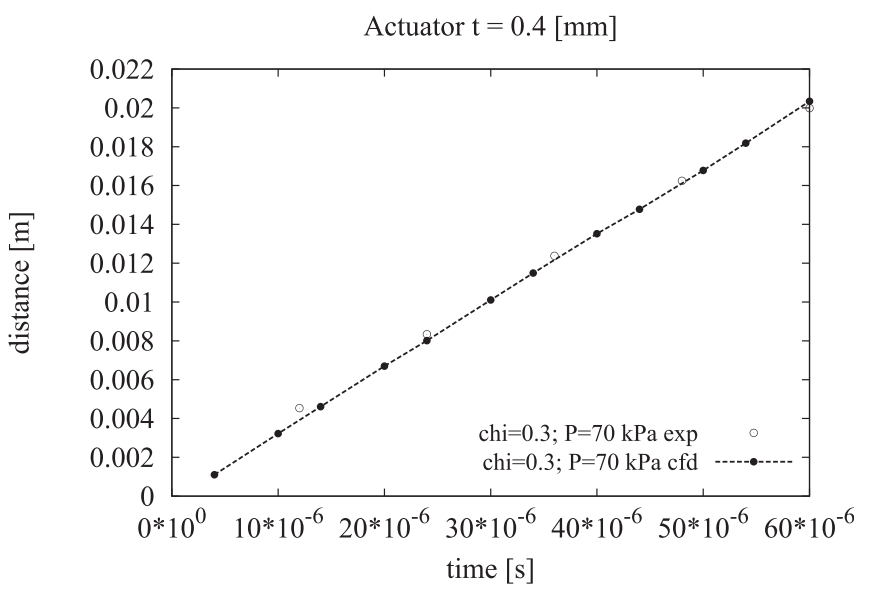

FIG. 21. Comparison of experimental and numerical shock wave propagation for an ambient pressure of $70 \mathrm{kPa}$.

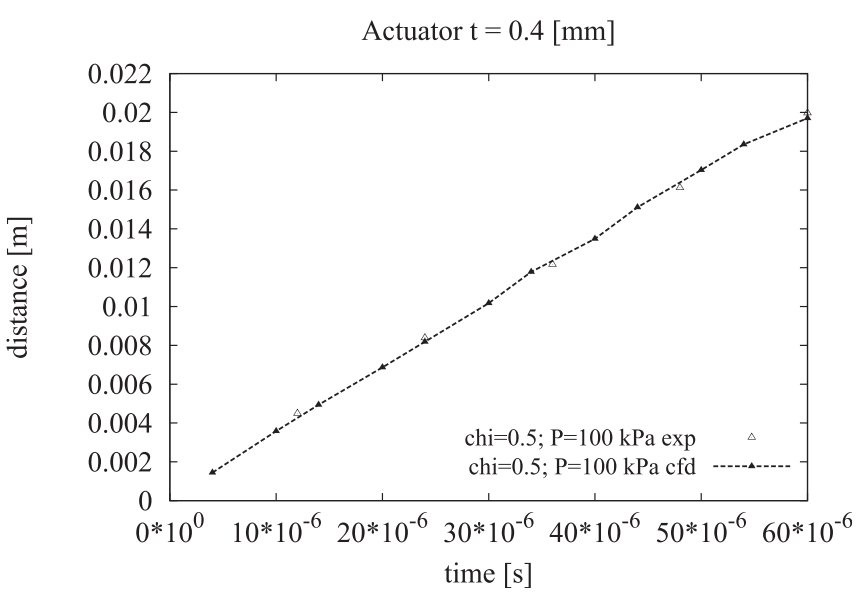

FIG. 22. Comparison of experimental and numerical shock wave propagation for an ambient pressure of $100 \mathrm{kPa}$. 
TABLE I. Temperature values added to the near wall region for the simulation of nsDBD generated shock waves. Temperature values corresponding to $\chi$ parameters used for CFD.

\begin{tabular}{lccc}
\hline \hline Pressure $(\mathrm{kPa})$ & $\chi$ & $\mathrm{T} \max (\mathrm{K})$ & $\mathrm{T}$ avg $(\mathrm{K})$ \\
\hline 30 & 0.1 & 410.319 & 295.214 \\
70 & 0.3 & 654.657 & 299.642 \\
100 & 0.5 & 898.995 & 304.071 \\
\hline \hline
\end{tabular}

\section{ACKNOWLEDGMENTS}

The authors are grateful to the Engineering and Physical Sciences Research Council (EPSRC) for their sponsorship of the Ph.D. project (Grant Nos. 2015/2016 EPSRC EP/M506539/1 ENG 70374 and in 2017 EPSRC DTG 2015 EP/M508056/1 EN) and the National Wind Tunnel Facility (EPSRC Grant No. EP/L024888/1) at the University of Glasgow. Special thanks are directed toward Professor Lucas for providing access to the high speed camera used.

\section{REFERENCES}

${ }^{1}$ T. C. Corke, C. L. Enloe, and S. P. Wilkinson, "Dielectric barrier discharge plasma actuators for flow control," Annu. Rev. Fluid Mech. 42, 505-529 (2010).

${ }^{2} \mathrm{G}$. Correale, "Preliminary investigation on ns-dbd plasma actuator for active flow separation control," Master's thesis, TU Delft, 2011.

${ }^{3} \mathrm{~K}$. Kourtzanidis and L. L. Raja, "Three electrode sliding nanosecond dielectric barrier discharge actuator: Modeling and physics," AIAA J. 55(4), 1393-1404 (2017).

${ }^{4}$ T. Unfer and J. P. Boeuf, "Modelling of a nanosecond surface discharge actuator," J. Phys. D: Appl. Phys. 42(19), 194017 (2009).

${ }^{5}$ J. Little, K. Takashima, M. Nishihara, I. Adamovich, and M. Samimy, "Separation control with nanosecond-pulse-driven dielectric barrier discharge plasma actuators," AIAA J. 50(2), 350-365 (2012).

${ }^{6} \mathrm{G}$. Correale, T. Michelis, D. Ragni, M. Kotsonis, and F. Scarano, "Nanosecondpulsed plasma actuation in quiescent air and laminar boundary layer," J. Phys. D: Appl. Phys. 47, 105201 (2014).

${ }^{7}$ D. V. Roupassov, A. A. Nikipelov, M. M. Nudnova, and A. Y. Starikovskii, "Flow separation control by plasma actuator with nanosecond pulsed-periodic discharge," AIAA J. 47(1), 168-185 (2009).

${ }^{8}$ D. Z. Pai, D. A. Lacoste, and C. O. Laux, "Nanosecond repetitively pulsed discharges in air at atmospheric pressure - the spark regime," Plasma Sources Sci. Technol. 19, 065015 (2010).

${ }^{9}$ M. S. Bak, W. Kim, and M. Cappelli, "On the quenching of excited electronic states of molecular nitrogen in nanosecond pulsed discharges in atmospheric pressure air," Appl. Phys. Lett. 98, 011502 (2011).

${ }^{10}$ A. Lo, G. Cleon, P. Vervisch, and A. Cessou, "Spontaneous raman scattering: A useful tool for investigating the afterglow of nanosecond scale discharges in air," Appl. Phys. B 107, 229-242 (2012).

${ }^{11}$ D. L. Rusterholtz, D. A. Lacoste, G. D. Stancu, D. Z. Pai, and C. O. Laux, "Ultrafast heating and oxygen dissociation in atmospheric pressure air by nanosecond repetitively pulsed discharges," J. Phys. D: Appl. Phys. 46, 464010 (2013).

${ }^{12}$ T. Michelis, G. Correale, I. B. Popov, M. Kotsonis, D. Ragni, S. Hulshoff, and L. Veldhuis, "Disturbance introduced into a laminar boundary layer by ns-dbd plasma actuator,” AIAA Paper No. 2013-0752, 2013.

${ }^{13}$ D. V. Roupassov, A. A. Nikipelov, M. M. Nudnova, and A. Y. Starikovskii, "Boundary layer separation control by nanosecond plasma actuator," in Proceedings of the 44th AIAA/ASME/SAE/ASEE Joint Propulsion Conference and Exhibit, Hartford, CT, July 2008.
${ }^{14}$ A. Russell, H. Zare-Behtash, and K. Kontis, "Characterisation of ns-dbd plasma actuators for supersonic flow control," in Proceedings of the 30th International Council of the Aeronautical Sciences, 2016.

${ }^{15}$ J. Van den Broecke, G. Correale, and F. Avallone, "Characteristics of efficiency if energy deposition of a ns-dbd plasma actuator,” AIAA Paper No. 2016-1212, 2016.

${ }^{16} \mathrm{~F}$. Avallone and G. Correale, "Method to quantify the electrical efficiency of a ns-dbd plasma actuator," in Proceedings of the Pacific Symposium on Flow Visualisation and Image Processing, 2015, Vol. 10, pp. 1-10.

${ }^{17} \mathrm{~N}$. A. Popov, "Investigation of the mechanism for rapid heating of nitrogen and air in gas discharges," Plasma Phys. Rep. 27(10), 886-896 (2001).

${ }^{18} \mathrm{~K}$. Takashima, Y. Zuzeek, W. Lempert, and I. Adamovich, "Characterization of a surface dielectric barrier discharge plasma sustained by repetitive nanosecond pulses," Plasma Sources Sci. Technol. 20, 055009 (2011).

${ }^{19}$ Z. Zhao, J. Li, J. Zheng, Y. D. Cui, and B. C. Khoo, "Study of shock and induced flow dynamics by nanosecond dielectric-barrier-discharge plasma actuators," AIAA J. 53(5), 1336-1348 (2015).

${ }^{20}$ Y. Liu, C. Kolbakir, H. Hu, A. Y. Starikovskiy, and R. Miles, “An experimental investigation on the thermal effects of ns-dbd and ac-dbd plasma actuators for aircraft icing mitigation," Plasma Sources Sci. Technol. 20, 1 (2019).

${ }^{21}$ J. Chen, H. Liang, Y. Wu, B. Wei, G. Zhao, M. Tian, and L. Xie, "Experimental study on anti-icing performance of ns-dbd plasma actuator," Appl. Sci. 8(10), 1889 (2018).

${ }^{22} \mathrm{R}$. Winkel, "Experimental study on the energy deposition of an ns-dbd plasma actuator and its effect on a laminar boundary layer," Master's thesis, TU Delft, 2015.

${ }^{23}$ F. do Nascimento, S. Moshkalev, and M. Machida, "Experimental analysis of dbd plasma jet properties using different gases and two kinds of transfer plate," e-print arXiv: 1512.03712.

${ }^{24}$ A. V. Nastuta, V. Pohoata, I. Mihaila, and I. Topala, "Diagnosis of a shortpulse dbd at atmospheric pressure in helium with hydrogen-methane admixtures," Phys. Plasmas 25(4), 043515 (2018).

${ }^{25}$ Y. Liu, Y. Hao, and B. Zheng, "Plasma formation in atmospheric pressure helium discharges under different background air pressures," Phys. Plasmas 19(9), 093506 (2012).

${ }^{26}$ T. Ukai, A. Russell, H. Zare-Behtash, and K. Kontis, "Temporal variation of the spatial density distribution above a nanosecond pulsed dielectric barrier discharge plasma actuator in quiescent air," Phys. Fluids 30, 116106 (2018).

${ }^{27} \mathrm{~J}$. A. Valerioti and T. C. Corke, "Pressure dependence of dielectric barrier discharge plasma flow actuators," AIAA J. 50, 1490-1502 (2012).

${ }^{28}$ N. Benard, N. Balcon, and E. Moreau, "Electric wind produced by a surface dielectric barrier discharge operating in air at different pressures: Aeronautical control insights," J. Phys. D: Appl. Phys. 41(4), 042002 (2008).

${ }^{29} \mathrm{P}$. Versailles, V. Gingras-Gosselin, and H. Vo, "Impact of pressure and temperature on the performance of plasma actuators," AIAA J. 48(4), 859-863 (2010).

${ }^{30} \mathrm{C}$. Y. Schuele and T. Corke, "Characteristics of single dielectric barrier discharge plasma actuators at subatmospheric pressures," in Proceedings of the 61st Annual American Physical Society Fluid Dynamics Meeting, San Antonio, Texas, November 2008.

${ }^{31}$ R. A. Dawson and J. Little, "Effects of pulse polarity on nanosecond pulse driven dielectric barrier discharge plasma actuators," J. Appl. Phys. 115, 043306 (2014).

${ }^{32}$ D. V. Gaitonde and M. H. McCrink, "A semi-empirical model of a nanosecond pulsed plasma actuator for flow control simulations with les," AIAA Paper No. 2012-0184, 2012.

${ }^{33}$ D. V. Gaitonde, "Analysis of plasma-based flow control mechanisms through large-eddy simulations,” Comput. Fluids 85, 19-26 (2013).

${ }^{34} \mathrm{~K}$. Kinefuchi, A. Y. Starikovskiy, and R. B. Miles, "Numerical investigation of nanosecond pulsed plasma actuators for control of shock-wave/boundary-layer separation," Phys. Fluids 30(10), 106105 (2018).

${ }^{35}$ K. Takashima, Z. Y. Yin, and I. V. Adamovich, "Measurements and kinetic modeling of energy coupling in volume and surface nanosecond pulse discharges," Plasma Sources Sci. Technol. 22, 015013 (2013).

${ }^{36}$ J. G. Zheng, Z. J. Zhao, J. Li, Y. D. Cui, and B. C. Khoo, "Numerical simulation of nanosecond pulsed dielectric barrier discharge actuator in a quiescent flow," Phys. Fluids 26(3), 036102 (2014). 